Research Article

Tamer Nabil*

\title{
Existence results for nonlinear coupled system of integral equations of Urysohn Volterra-Chandrasekhar mixed type
}

https://doi.org/10.1515/dema-2020-0017

received March 26, 2020; accepted July 22, 2020

Abstract: The combined systems of integral equations have become of great importance in various fields of sciences such as electromagnetic and nuclear physics. New classes of the merged type of Urysohn Volterra-Chandrasekhar quadratic integral equations are proposed in this paper. This proposed system involves fractional Urysohn Volterra kernels and also Chandrasekhar kernels. The solvability of a coupled system of integral equations of Urysohn Volterra-Chandrasekhar mixed type is studied. To realize the existence of a solution of those mixed systems, we use the Perov's fixed point combined with the LeraySchauder fixed point approach in generalized Banach algebra spaces.

Keywords: fixed point, generalized Banach algebra, Chandrasekhar's integral equation, Urysohn-Volterra integral equation

MSC 2020: 47H10, 45G15

\section{Introduction}

Integral equations are an important topic in functional analysis (see, [1-3], for examples). They are stratified in the characterization of many real life events such as processes encountered in nuclear physics [4], heat conduction [5], electromagnetic [6] and multimedia processing [7].

Among the many integral equations that were established in mathematical analysis and were stratified to many areas of engineering and real life sciences, an efficient and effective role is played by integral equations of fractional kernels [8-11].

Fractional calculus is an essential and useful branch of mathematical analysis that investigates derivatives and integrals of fractional order. A long time ago, there were many definitions for fractional integral operators, such as Riemann-Liouville, Hadamard, Katugampola and Erdelyi-Kober fractional integral operators. Recently, in 2017, Almeida [12] proposed a new definition of the fractional integral and called this operator $\psi$-Caputo integral. This new definition is more generalized than Riemann-Liouville, Hadamard, Erdelyi Kober and Caputo operator kinds. Suppose that $C^{n}(I, \mathbb{R}), n \in \mathbb{N}$, is the space of all $n$-times continuous and differentiable functions from $I=[0, a]$ to $\mathbb{R}$. Let $\psi \in C^{1}(I, \mathbb{R})$ be an increasing function. Let $u: I \rightarrow \mathbb{R}$ be an integrable function. The $\psi$-Riemann-Liouville fractional integral of order $\alpha>0, \alpha \in \mathbb{R}$ of the function $u$ is defined as

$$
J_{0^{+}}^{\alpha, \psi} u(t)=\frac{1}{\Gamma(\alpha)} \int_{0}^{t} \psi^{\prime}(\zeta)(\psi(t)-\psi(\zeta))^{\alpha-1} u(\zeta) \mathrm{d} \zeta,
$$

\footnotetext{
* Corresponding author: Tamer Nabil, Suez Canal University, Faculty of Computers and Informatics, Department of Basic Science, Ismailia, Egypt, e-mail: t_3bdelsadek@yahoo.com
} 
where $n=[\alpha]+1$ and $[\alpha]$ denotes the integral part of $\alpha$.

In 2018, Darwish et al. [13] applied the approach of Darbo's fixed point to investigate the following Urysohn-Volterra integral equation:

$$
u(t)=f(t, u(t))+g(t, u(t)) \int_{0}^{t} \frac{\psi^{\prime}(s)(\psi(t)-\psi(s))^{p-1}}{\Gamma(p)} h(s, u(s)) \mathrm{d} s, \quad t \in[0, a],
$$

where $(a>0) \in \mathbb{R}_{+}$and $p \in(0,1)$. The authors obtained the existence results of the solution of equation (1) under some certain conditions. In the same year, Nieto et al. [14] proposed some new versions of the fixed point theorems in the algebra of generalized Banach spaces. They established the type of Krasnoselskii and Leray-Schauder fixed point for the product and sum of more than or equal two operators.

In 2019, Hashem et al. [15] applied a fixed point approach according to Amar et al. [16] to study the following system:

$$
\begin{aligned}
& u(t)=\mathcal{F}_{1}(t, u(t))+\mathcal{G}_{1}(t, v(t)) \int_{0}^{t} \frac{(t-s)^{p-1}}{\Gamma(p)} \mathcal{S}_{1}(s, v(s)) \mathrm{d} s, \quad t \in[0, a], \\
& v(t)=\mathcal{F}_{2}(t, v(t))+\mathcal{G}_{2}(t, u(t)) \int_{0}^{t} \frac{(t-s)^{q-1}}{\Gamma(q)} \mathcal{S}_{2}(s, u(s)) \mathrm{d} s, \quad t \in[0, a],
\end{aligned}
$$

where $(a>0) \in \mathbb{R}_{+}$and $p, q \in(0,1)$.

One of the interesting types of integral equations is the so-called Chandrasekhar integral equation. The integral equation of Chandrasekhar's integral equation is considered by Chandrasekhar [17] to model the process of radiative transfer. From this date onward, this type has attracted a lot of attention from many researchers [18-21].

In 1998, Banas et al. [18] investigated the solvability of the following quadratic Chandrasekhar integral equation:

$$
u(t)=1+t u(t) \int_{0}^{1} \frac{f(s)}{t+s} u(s) \mathrm{d} s
$$

where $t \in[0,1]$ and $f:[0,1] \rightarrow \mathbb{R}$ is continuous. Recently, in 2017, Hashem and El-Sayed [19] considered the following two-dimensional quadratic Chandrasekhar integral equations:

$$
\begin{aligned}
& u(t)=a_{1}(t)+g_{1}(t, v(t)) \int_{0}^{t} \frac{t}{t+s} f_{1}(s, v(s)) \mathrm{d} s, \quad t \in[0, a], \\
& v(t)=a_{2}(t)+g_{2}(t, u(t)) \int_{0}^{t} \frac{t}{t+s} f_{1}(s, u(s)) \mathrm{d} s, \quad t \in[0, a] .
\end{aligned}
$$

They proved that system (4) has at least one continuous solution by applying the Schauder fixed point theorem. In the same year, Hashem [22] investigated a more generalized coupled system of Chandrasekhar integral equations which is given by

$$
\begin{aligned}
& u(t)=\mathcal{A}_{1}(t, u(t))+g_{1}(t, v(t)) \int_{0}^{t} \frac{t}{t+s} f_{1}(s, v(s)) \mathrm{d} s, \quad t \in[0, a], \\
& v(t)=\mathcal{A}_{2}(t, v(t))+g_{2}(t, u(t)) \int_{0}^{t} \frac{t}{t+s} f_{2}(s, u(s)) \mathrm{d} s, \quad t \in[0, a] .
\end{aligned}
$$

The existence of a solution of system (5) was shown via a block operator $(2 \times 2)$-matrix approach which was proposed by Jeribi et al. [23]. In 2018, Chang and Feng [20] reviewed many results and applications for Chandrasekhar integral equations. 
The solvability of quadratic integral equations has been established in many papers; see, for example, [24,25]. More recently, in 2019, Jeribi et al. [21] investigated the solvability of the following coupled system of Chandrasekhar functional integral equations:

$$
\begin{aligned}
& u(t)=\mathcal{A}_{1}(t, u(t))+g_{1}(t, v(t)) \int_{0}^{t} \frac{t}{t+s} f_{1}(s, v(s)) \mathrm{d} s, \quad t \in[0,1], \\
& v(t)=\mathcal{A}_{2}(t, v(t))+\Phi\left(t, \int_{0}^{t} \frac{t}{t+s} f_{2}(s, u(s)) \mathrm{d} s\right), \quad t \in[0,1] .
\end{aligned}
$$

In the next year, Jeribi et al. [26] applied a fixed point approach for a $2 \times 2$ block operator matrix to study the solvability for infinite system of integral equations.

The merged systems of integral equations have become of great importance now in various fields of science such as electromagnetic and nuclear physics [4,6]. In this paper, we propose the more general coupled system of Urysohn Volterra-Chandrasekhar integral equations, given by

$$
\begin{aligned}
& u(t)=\left(p_{1}(t)+J_{0^{+}}^{\alpha, \psi} V_{1}(t, u(s), v(t))\right) \cdot\left(q_{1}(t)+H_{1}(t, u(t), v(t)) \int_{0}^{t} \frac{t}{t+s} F_{1}(s, u(s), v(s)) \mathrm{d} s\right), \quad t \in I=[0,1], \\
& v(t)=\left(p_{2}(t)+J_{0^{+}}^{\beta, \psi} V_{2}(t, u(s), v(t))\right) \cdot\left(q_{2}(t)+H_{2}(t, u(t), v(t)) \int_{0}^{t} \frac{t}{t+s} F_{2}(s, u(s), v(s)) \mathrm{d} s\right), \quad t \in I=[0,1],
\end{aligned}
$$

where $\alpha$ and $\beta \in(0,1)$. It is clear that the proposed system is more general and comprehensive than systems (4), (5) and (6). Also, all functions in the proposed system involve the unknowns $u, v$, which is an advantage to system (7).

This manuscript is organized as follows: Section 2 is devoted to giving some facts, basic results and definitions which are used in the results. In Section 3, we study the solvability of system (7).

\section{Basic concepts and auxiliary facts}

Throughout this paper, $\mathbb{R}_{+}^{n}$ is the set $\left\{\Pi=\left(\varpi_{1}, \ldots, \varpi_{n}\right) \in \mathbb{R}^{n}: \varpi_{i}>0 \quad \forall i=1,2, \ldots, n\right\}$. Let $\Lambda=\left(\varrho_{1}, \ldots, \varrho_{n}\right) \in \mathbb{R}^{n}$. Let $\preccurlyeq_{n}$ be a partial order on $\mathbb{R}^{n}$ such that: $\Pi \preccurlyeq_{n} \Lambda \Leftrightarrow \varpi_{i} \leq \varrho_{i}$ for all $i=1,2, \ldots, n$. Therefore, if $\delta \in \mathbb{R}$, then $\Pi \preccurlyeq_{n} \delta$ means $\varpi_{i} \leq \delta$ for all $i=1,2, \ldots, n$. Also, $|\Pi|=\left(\left|\varpi_{1}\right|, \ldots,\left|\varpi_{n}\right|\right)$ and $\max (\Pi, \Lambda)=\left(\max \left\{\varpi_{1}, \varrho_{1}\right\}, \ldots\right.$, $\left.\max \left\{\varpi_{n}, \varrho_{n}\right\}\right)$. Furthermore, consider $0_{n}$ to be the zero of $\mathbb{R}^{n}$. Next, we give the concept of generalized metric space. For more details see [14,27-30].

Definition 1. [30] Let $X \neq \varphi$ and $\rho: X \times X \rightarrow \mathbb{R}^{n}$. Then $\rho$ is said to be a vector-valued-metric on $X$ if for all $\left(\Lambda_{1}, \Lambda_{2}, \Lambda_{3}\right) \in X^{3}$ the following conditions hold:

(1) $\rho\left(\Lambda_{1}, \Lambda_{2}\right) \succcurlyeq_{n} 0_{n}$;

(2) $\rho\left(\Lambda_{1}, \Lambda_{2}\right)=0_{n} \Leftrightarrow \Lambda_{1}=\Lambda_{2}$;

(3) $\rho\left(\Lambda_{1}, \Lambda_{2}\right)=\rho\left(\Lambda_{2}, \Lambda_{1}\right)$;

(4) $\rho\left(\Lambda_{1}, \Lambda_{2}\right) \preccurlyeq_{n} \rho\left(\Lambda_{1}, \Lambda_{3}\right)+\rho\left(\Lambda_{3}, \Lambda_{2}\right)$.

$(X, \rho)$ is called a generalized metric space and $\rho$ is given by

$$
\rho\left(\Lambda_{1}, \Lambda_{2}\right)=\left(\begin{array}{c}
\rho_{1}\left(\Lambda_{1}, \Lambda_{2}\right) \\
\rho_{2}\left(\Lambda_{1}, \Lambda_{2}\right) \\
\vdots \\
\rho_{n}\left(\Lambda_{1}, \Lambda_{2}\right)
\end{array}\right)
$$


Clearly, $\rho$ is a generalized metric on $X$ if and only if $\rho_{i}$ is a metric in the usual sense, for all $i=1,2, \ldots, n$. We indicate that the definitions of sequences, Cauchy sequences, convergence, closed and open subsets and completeness are the same as those for usual metric spaces.

Definition 2. [14] Let $X$ be a vector space over $\mathbb{R}$. A vector-valued generalized norm on $X$ is $\|\cdot\|: X \rightarrow \mathbb{R}_{+}^{n}$ such that for all $\left(\Lambda_{1}, \Lambda_{2}\right) \in X$ and $\kappa \in \mathbb{R}$, we have that:

(1) $\left\|\Lambda_{1}\right\| \geqslant_{n} 0_{n}$

(2) $\left\|\Lambda_{1}\right\|=0_{n} \Leftrightarrow \Lambda_{1}=0_{n}$;

(3) $\left\|\kappa \Lambda_{1}\right\|=|\kappa||| \Lambda_{1}||$;

(4) $\left\|\Lambda_{1}+\Lambda_{2}\right\| \leqslant_{n}\left\|\Lambda_{1}\right\|+\left\|\Lambda_{2}\right\|$.

$(X,\|\cdot\|)$ is said to be a generalized normed space. If $\left(\rho\left(\Lambda_{1}, \Lambda_{2}\right)=\left\|\Lambda_{1}-\Lambda_{2}\right\|\right)$ is complete, then $(X,\|\cdot\|)$ is said to be a generalized Banach space.

Definition 3. [14] Let $\mathcal{M}_{n \times n}\left(\mathbb{R}_{+}\right)=\left\{L=\left(\alpha_{i j}\right)_{i, j=1, \ldots, n}: \alpha_{i j} \in \mathbb{R}_{+}, \forall i, j=1, \ldots n\right\}$. Suppose that $L \in \mathcal{M}_{n \times n}\left(\mathbb{R}_{+}\right)$. Then the spectral radius $\sigma(L)$ of $L$ is defined as

$$
\sigma(L)=\max \left\{\left|\mu_{j}(L)\right|: i=1,2, \ldots, N\right\},
$$

where $\mu_{j}(L), i=1, \ldots, n$, are the eigenvalues for $L$.

Definition 4. [14] The generalized Banach algebra $X$ is an algebra which is also a generalized Banach space, such that for all $\Lambda_{1}, \Lambda_{2} \in X$, the following holds:

$$
\left\|\Lambda_{1} \Lambda_{2}\right\| \preccurlyeq_{n}\left\|\Lambda_{1}\right\|\left\|\Lambda_{2}\right\|,
$$

where

$$
\left\|\Lambda_{1} \Lambda_{2}\right\|=\left(\begin{array}{c}
\left\|\Lambda_{1} \Lambda_{2}\right\|_{1} \\
\left\|\Lambda_{1} \Lambda_{2}\right\|_{2} \\
\vdots \\
\left\|\Lambda_{1} \Lambda_{2}\right\|_{n}
\end{array}\right)
$$

and

$$
\left\|\Lambda_{1}\right\|\left\|\Lambda_{2}\right\|=\left(\begin{array}{cc}
\left\|\Lambda_{1}\right\|_{1} & \left\|\Lambda_{2}\right\|_{1} \\
\left\|\Lambda_{1}\right\|_{2} & \left\|\Lambda_{2}\right\|_{2} \\
& \vdots \\
\left\|\Lambda_{1}\right\|_{n} & \left\|\Lambda_{2}\right\|_{n}
\end{array}\right) .
$$

Definition 5. [30] Suppose that $(X, \rho)$ is a generalized metric space. The map $\Phi: X \rightarrow X$ is called contractive (or a contraction) if there exists an $L \in \mathcal{M}_{n \times n}\left(\mathbb{R}_{+}\right)$such that $\sigma(L)<1$ and

$$
\rho\left(\Phi \Lambda_{1}, \Phi \Lambda_{2}\right) \preccurlyeq_{N} L \rho\left(\Lambda_{1}, \Lambda_{2}\right),
$$

for all $\left(\Lambda_{1}, \Lambda_{2}\right) \in X^{2}$.

The following theorem, due to Nieto et al. [14], combines Leray-Schauder with Perov's fixed point approach in generalized vectorial algebra Banach spaces and is used to prove our results.

Theorem 6. [14] Let $X$ be a generalized Banach algebra, $0 \in \Omega$ be an open, bounded and convex subset of $X$. Suppose that $\mathcal{A}: \Omega \rightarrow X, \mathcal{B}: \bar{\Omega} \rightarrow X$ are such that

(1) $\mathcal{A}$ is an $L$ contraction, where $L \in \mathcal{M}_{n \times n}\left(\mathbb{R}_{+}\right)$and $L=\left(\alpha_{i j}\right)_{1 \leq i, j \leq n}$;

(2) $\mathcal{B}$ is a completely continuous; 
(3) for all $y \in X,\left(\frac{I_{X}}{\mathcal{A}}\right)^{-1}$ exists on $\mathcal{B}(\bar{\Omega})$, where $I_{X}(y)=y$ and $\frac{I_{X}}{\mathcal{A}}: X \rightarrow X$ is defined as $\left(\frac{I_{X}}{\mathcal{A}}\right)(y)=\frac{y}{\mathcal{A}(y)}$;

(4) If

$$
\left(\begin{array}{c}
l_{1} \\
l_{2} \\
\vdots \\
l_{n}
\end{array}\right) \geqslant_{n} \quad \sup _{\omega \in \bar{\Omega}}\|\mathcal{B}(\omega)\|
$$

and

$$
L^{*}=\left(\begin{array}{ccc}
l_{1} \alpha_{11} & \ldots & l_{n} \alpha_{1 n} \\
\vdots & \ddots & \vdots \\
l_{1} \alpha_{n 1} & \ldots & l_{n} \alpha_{n n}
\end{array}\right),
$$

then $L^{*} \in \mathcal{M}_{n \times n}\left(\mathbb{R}_{+}\right)$and $\sigma\left(L^{*}\right)<1$.

Then either:

(I) the equation $\omega=\mathcal{A}(\omega) \mathcal{B}(\omega)$ has a solution in $\bar{\Omega}$, or

(II) there exists $u \in \bar{\Omega} \backslash \Omega$ such that

$$
u=\lambda \mathcal{A}\left(\frac{u}{\lambda}\right) \mathcal{B}(u), \quad \lambda \in(0,1)
$$

\section{Existence theory}

The investigation of solvability of system (7) is given under the following conditions.

(C1) The functions $V_{i}: I \times \mathbb{R}^{2} \rightarrow \mathbb{R}, i=1,2$ are continuous in $t \in I$, for any $(u, v) \in \mathbb{R}^{2}$ and continuous in $u, v \in \mathbb{R}$ for all $t \in I$. There exists $p_{i j} \in \mathbb{R}_{+}, i, j=1,2$ such that for $i=1,2$, we have

$$
\left|V_{i}\left(t, x_{1}, x_{2}\right)-V_{i}\left(t, y_{1}, y_{2}\right)\right| \leq \sum_{j=1}^{2} p_{i j}\left|x_{i}-y_{i}\right|
$$

for all $t \in I$ and $\left(x_{1}, x_{2}, y_{1}, y_{2}\right) \in \mathbb{R}^{4}$.

(C2) The functions $F_{i}: I \times \mathbb{R}^{2} \rightarrow \mathbb{R}, i=1,2$ are measurable in $t$ for all $u, v \in \mathbb{R}$ and continuous in $u, v \in \mathbb{R}$ for almost all $t \in I$ such that

$$
\left|F_{i}(t, u, v)\right| \leq f_{i}(t) \in L^{1}(I), \quad i=1,2,
$$

for all $(t, u, v) \in I \times \mathbb{R}^{2}$ and

$$
K_{i}=\sup _{t \in I} \int_{0}^{1} \frac{1}{t+s}\left|f_{i}(s)\right| \mathrm{d} s, \quad i=1,2 .
$$

(C3) The functions $H_{i}: I \times \mathbb{R}^{2} \rightarrow \mathbb{R}, i=1,2$ are continuous and

$$
N_{j}=\max _{(s, u, v) \in I \times \mathbb{R}^{2}}\left|H_{i}(s, u, v)\right|, \quad i=1,2 .
$$

(C4) The mappings $p_{i}, q_{i}: I \rightarrow \mathbb{R}, i=1,2$ are continuous and there exists $W^{p_{i}}, W^{q_{i}}, i=1,2$ such that

$$
\begin{array}{ll}
W^{p_{i}} & =\sup _{t \in I}\left|p_{i}(t)\right|, \quad i=1,2, \\
W^{q_{i}} & =\sup _{t \in I}\left|q_{i}(t)\right|, \quad i=1,2 .
\end{array}
$$


(C5) $\sigma(Q)<1$, where $Q=\left(\gamma_{i j}\right)_{i=1,2, j=1,2}$ where

$$
\begin{aligned}
& \gamma_{1 i}=\frac{(\psi(1)-\psi(0))^{\alpha}}{\Gamma(\alpha+1)} p_{1 i}, \quad i=1,2, \\
& \gamma_{2 i}=\frac{(\psi(1)-\psi(0))^{\beta}}{\Gamma(\beta+1)} p_{2 i}, \quad i=1,2 .
\end{aligned}
$$

(C6) Let $p^{*}=\max \left\{\frac{(\psi(1)-\psi(0))^{\alpha}}{\Gamma(\alpha+1)} p_{1 j}, \frac{(\psi(1)-\psi(0))^{\beta}}{\Gamma(\beta+1)} p_{2 j}, j=1,2\right\}$, then the inequality $p^{*}\left(W^{q_{1}}+N_{1} K_{1}+W^{q_{2}}+N_{2} K_{2}\right)<1$ holds.

Let $C(I)$ be the Banach algebra of all continuous real-valued functions on $I$ with the supremum norm $\|u\|_{\infty}=\sup _{t \in I}|u(t)|$, for all $u \in C(I)$ and with respect to the pointwise product of functions. Now, let $X=$ $C(I) \times C(I)$. Define the generalized norm $\|\cdot\|: X \rightarrow \mathbb{R}_{+}^{2}$ as

$$
\|(u, v)\|=\left(\begin{array}{l}
\|u\|_{\infty} \\
\|v\|_{\infty}
\end{array}\right)
$$

for all $(u, v) \in X$. Clearly $(X,\|\cdot\|)$ is generalized Banach algebra. Let $d: X \times X \rightarrow \mathbb{R}_{+}^{2}$ be the generalized metric space induced by the norm which is defined as

$$
d\left(\left(u_{1}, v_{1}\right),\left(u_{2}, v_{2}\right)\right)=\left(\begin{array}{c}
\left\|u_{1}-u_{2}\right\|_{\infty} \\
\left\|v_{1}-v_{2}\right\|_{\infty}
\end{array}\right),
$$

for all $\left(u_{1}, v_{1}\right),\left(u_{2}, v_{2}\right) \in X$.

Note system $(7)$ can be written as $(u, v)=\mathcal{A}(u, v) \mathcal{B}(u, v)$. The operator $\mathcal{A}(x, y)=\left(\mathcal{A}_{1}(u, v), \mathcal{A}_{2}(u, v)\right)$, where

$$
\begin{aligned}
& {\left[\mathcal{A}_{1}(u, v)\right](t)=p_{1}(t)+\int_{0}^{t} \frac{\psi^{\prime}(s)(\psi(t)-\psi(s))^{\alpha-1}}{\Gamma(\alpha)} V_{1}(s, u(s), v(s)) \mathrm{d} s, \quad t \in I,} \\
& {\left[\mathcal{A}_{2}(u, v)\right](t)=p_{2}(t)+\int_{0}^{t} \frac{\psi^{\prime}(s)(\psi(t)-\psi(s))^{\beta-1}}{\Gamma(\beta)} V_{2}(s, u(s), v(s)) \mathrm{d} s, \quad t \in I .}
\end{aligned}
$$

The operator $\mathcal{B}$ is the Chandrasekhar integral operator and has the form $\mathcal{B}(x, y)=\left(\mathcal{B}_{1}(u, v), \mathcal{B}_{2}(u, v)\right)$, where the superposition operators $\mathcal{B}_{1}, \mathcal{B}_{2}$ are defined as:

$$
\left[\mathcal{B}_{1}(u, v)\right](t)=q_{1}(t)+H_{1}(t, u(t), v(t)) \int_{0}^{t} \frac{t}{t+s} F_{1}(s, u(s), v(s)) \mathrm{d} s, \quad t \in I
$$

and

$$
\left[\mathcal{B}_{2}(u, v)\right](t)=q_{2}(t)+H_{2}(t, u(t), v(t)) \int_{0}^{t} \frac{t}{t+s} F_{2}(s, u(s), v(s)) \mathrm{d} s, \quad t \in I .
$$

Define $\mathbb{T}: C(I) \times C(I) \rightarrow X$ as:

$$
\mathbb{T}(u, v)=\mathcal{A}(u, v) \mathcal{B}(u, v) .
$$

Clearly, the solution of system (7) is the fixed point of the operator $\mathbb{T}$.

Lemma 7. Under assumptions (C1)-(C6), the operator $\mathcal{A}$ is a contractive mapping. 
Proof. Let $\left(u_{1}, v_{1}\right),\left(u_{2}, v_{2}\right) \in X$, then we get

$$
\begin{aligned}
\left|\left[\mathcal{A}_{1}\left(u_{1}, v_{1}\right)\right](t)-\left[\mathcal{A}_{1}\left(u_{2}, v_{2}\right)\right](t)\right|= & \mid \int_{0}^{t} \frac{\psi^{\prime}(s)(\psi(t)-\psi(s))^{\alpha-1}}{\Gamma(\alpha)} V_{1}\left(s, u_{1}(s), v_{1}(s)\right) \mathrm{d} s \\
& -\int_{0}^{t} \frac{\psi^{\prime}(s)(\psi(t)-\psi(s))^{\alpha-1}}{\Gamma(\alpha)} V_{1}\left(s, u_{2}(s), v_{2}(s)\right) \mathrm{d} s \mid \\
\leq & \int_{0}^{t} \frac{\psi^{\prime}(s)(\psi(t)-\psi(s))^{\alpha-1}}{\Gamma(\alpha)} \mid V_{1}\left(s, u_{1}(s), v_{1}(s)-V_{2}\left(s, u_{2}(s), v_{2}(s)\right) \mid \mathrm{d} s\right. \\
& \leq \frac{(\psi(1)-\psi(0))^{\alpha}}{\Gamma(\alpha+1)}\left(p_{11}\left\|u_{1}-u_{2}\right\|_{\infty}+p_{12}\left\|v_{1}-v_{2}\right\|_{\infty}\right) .
\end{aligned}
$$

Hence, we have

$$
\left.\| \mathcal{A}_{1}\left(u_{1}, v_{1}\right)-\mathcal{A}_{1}\left(u_{2}, v_{2}\right)\right) \|_{\infty} \leq \frac{(\psi(1)-\psi(0))^{\alpha}}{\Gamma(\alpha+1)}\left(p_{11}\left\|u_{1}-u_{2}\right\|_{\infty}+p_{12}\left\|v_{1}-v_{2}\right\|_{\infty}\right) .
$$

Similarly, we get

$$
\left.\| \mathcal{A}_{2}\left(u_{1}, v_{1}\right)-\mathcal{A}_{2}\left(u_{2}, v_{2}\right)\right) \|_{\infty} \leq \frac{(\psi(1)-\psi(0))^{\beta}}{\Gamma(\beta+1)}\left(p_{21}\left\|u_{1}-u_{2}\right\|_{\infty}+p_{22}\left\|v_{1}-v_{2}\right\|_{\infty}\right) .
$$

So, we get that

$$
d\left(\mathcal{A}\left(u_{1}, v_{1}\right), \mathcal{A}\left(u_{2}, v_{2}\right)\right) \preccurlyeq_{2} Q\left(\begin{array}{l}
\left\|u_{1}-u_{2}\right\|_{\infty} \\
\left\|v_{1}-v_{2}\right\|_{\infty}
\end{array}\right)
$$

and $Q=\left(\gamma_{i j}\right)_{i=1,2, j=1,2}$, where

$$
\begin{aligned}
& \gamma_{1 i}=\frac{(\psi(1)-\psi(0))^{\alpha}}{\Gamma(\alpha+1)} p_{1 i}, \quad i=1,2, \\
& \gamma_{2 i}=\frac{(\psi(1)-\psi(0))^{\beta}}{\Gamma(\beta+1)} p_{2 i}, \quad i=1,2 .
\end{aligned}
$$

Since $\sigma(Q)<1, \mathcal{A}$ is a contractive operator.

Lemma 8. Suppose that assumptions (C1)-(C6) hold. Then, there exists $K^{*}$ such that, for every solution $(u, v) \in X$ of the following system:

$$
\begin{aligned}
& u=\lambda \mathcal{A}_{1}\left(\frac{u}{\lambda}, \frac{v}{\lambda}\right) \mathcal{B}_{1}(u, v), \\
& v=\lambda \mathcal{A}_{2}\left(\frac{u}{\lambda}, \frac{v}{\lambda}\right) \mathcal{B}_{2}(u, v),
\end{aligned}
$$

for some $\lambda \in(0,1)$, we get $\|u\|_{\infty} \leq K^{*}$ and $\|v\|_{\infty} \leq K^{*}$.

Proof. Let $(u, v) \in X$ be a solution of (15), then we have

$$
\begin{aligned}
\frac{u}{\lambda} & =\mathcal{A}_{1}\left(\frac{u}{\lambda}, \frac{v}{\lambda}\right) \mathcal{B}_{1}(u, v), \\
\frac{v}{\lambda} & =\mathcal{A}_{2}\left(\frac{u}{\lambda}, \frac{v}{\lambda}\right) \mathcal{B}_{2}(u, v) .
\end{aligned}
$$

For simplicity, suppose that $\hat{u}=\frac{u}{\lambda}$ and $\hat{v}=\frac{v}{\lambda}$. Then, we get 


$$
\begin{aligned}
|\hat{u}(t)| \leq & \left|\left[\mathcal{A}_{1}(\hat{u}, \hat{v})\right](t)\right|\left|\left[\mathcal{B}_{1}(\lambda \hat{u}, \lambda \hat{v})\right](t)\right| \\
\leq & \left.|| p_{1}(t)\left|+\int_{0}^{t} \frac{\psi^{\prime}(s)(\psi(t)-\psi(s))^{\alpha-1}}{\Gamma(\alpha)}\right| V_{1}(s, \hat{u}(s), \hat{v}(s)) \mid \mathrm{d} s\right) \\
& \left.\times\left|q_{1}(t)+H_{1}(t, \lambda \hat{u}(t), \lambda \hat{v}(t))\right| \int_{0}^{t} \frac{t}{t+s}\left|F_{1}(s, \lambda \hat{u}(s), \lambda \hat{v}(s))\right| \mathrm{d} s\right) \\
\leq & \left(W^{p_{1}}+\frac{(\psi(1)-\psi(0))^{\alpha}}{\Gamma(\alpha+1)}\left(p_{11}\|\hat{u}\|_{\infty}+p_{12}\|\hat{v}\|_{\infty}+\left|V_{1}(t, 0,0)\right|\right)\right)\left(W^{q_{1}}+N_{1} K_{1}\right) .
\end{aligned}
$$

Similarly, we have

$$
|\hat{v}(t)| \leq\left(W^{p_{2}}+\frac{(\psi(1)-\psi(0))^{\beta}}{\Gamma(\beta+1)}\left(p_{21}\|\hat{u}\|_{\infty}+p_{22}\|\hat{v}\|_{\infty}+\left|V_{2}(t, 0,0)\right|\right)\right)\left(W^{q_{2}}+N_{2} K_{2}\right) .
$$

Let

$$
A_{V}^{*}=\max \left\{\frac{(\psi(1)-\psi(0))^{\alpha}}{\Gamma(\alpha+1)}\left\|V_{1}(t, 0,0)\right\|_{\infty}, \frac{(\psi(1)-\psi(0))^{\beta}}{\Gamma(\beta+1)}\left\|V_{2}(t, 0,0)\right\|_{\infty}\right\}
$$

and

$$
p^{*}=\max \left\{\frac{(\psi(1)-\psi(0))^{\alpha}}{\Gamma(\alpha+1)} p_{1 j}, \frac{(\psi(1)-\psi(0))^{\beta}}{\Gamma(\beta+1)} p_{2 j}, j=1,2\right\} .
$$

Then we have

$$
|\hat{u}(t)| \leq\left(W^{p_{1}}+\frac{(\psi(1)-\psi(0))^{\alpha}}{\Gamma(\alpha+1)}\left(p^{*}\|\hat{u}\|_{\infty}+p^{*}\|\hat{v}\|_{\infty}+A_{V}^{*}\right)\left(W^{q_{1}}+N_{1} K_{1}\right) .\right.
$$

Therefore, we get

$$
|\hat{v}(t)| \leq W^{p_{2}}+\frac{(\psi(1)-\psi(0))^{\beta}}{\Gamma(\beta+1)}\left(p^{*}\|\hat{u}\|_{\infty}+p^{*}\|\hat{v}\|_{\infty}+A_{V}^{*}\right)\left(W^{q_{2}}+N_{2} K_{2}\right) .
$$

By adding the last two inequalities, we get

$$
\begin{aligned}
|\hat{u}(t)|+|\hat{v}(t)| \leq & \left(W^{p_{1}}+A_{V}^{*}\right)\left(W^{q_{1}}+N_{1} K_{1}\right)+\left(W^{p_{2}}+A_{V}^{*}\right)\left(W^{q_{2}}+N_{2} K_{2}\right) \\
& +p^{*}\left(\|\hat{u}\|_{\infty}+\|\hat{v}\|_{\infty}\right)\left(W^{q_{1}}+N_{1} K_{1}+W^{q_{2}}+N_{2} K_{2}\right) .
\end{aligned}
$$

Let $\mathbb{L}=p^{*}\left(W^{q_{1}}+N_{1} K_{1}+W^{q_{2}}+N_{2} K_{2}\right)$ and $\mathbb{K}=\left(W^{p_{1}}+A_{V}^{*}\right)\left(W^{q_{1}}+N_{1} K_{1}\right)+\left(W^{p_{2}}+A_{V}^{*}\right)\left(W^{q_{2}}+N_{2} K_{2}\right)$. Since $\mathbb{L}<1$, we get

$$
\|u\|_{\infty}+\|v\|_{\infty} \leq \frac{\mathbb{K}}{1-\mathbb{L}},
$$

which implies

$$
\|u\|_{\infty}+\|v\|_{\infty} \leq \frac{|\lambda| \mathbb{K}}{1-\mathbb{L}} \leq \frac{\mathbb{K}}{1-\mathbb{L}}=\mathbb{K}^{*} .
$$

Thus, we get $\|u\|_{\infty} \leq \mathbb{K}^{*}$ and $\|v\|_{\infty} \leq \mathbb{K}^{*}$.

Lemma 9. Let assumptions (C1)-(C6) hold. The operator $\mathcal{B}: \bar{\Omega} \rightarrow X$ is completely continuous.

Proof. The proof is obtained in three steps.

Step 1. $\mathcal{B}$ is continuous. Let $\left\{\left(u_{n}, v_{n}\right)\right\}$ be a sequence in $X$ such that $\left(u_{n}, v_{n}\right) \rightarrow(u, v) \in X$ as $n \rightarrow \infty$. Then we have $u_{n} \rightarrow u \in C(I)$ and $v_{n} \rightarrow v \in C(I)$ as $n \rightarrow \infty$. So, for all $t \in I$, we get 


$$
\begin{aligned}
& \left|\left[\mathcal{B}_{1}\left(u_{n}, v_{n}\right)\right](t)-\left[\mathcal{B}_{1}(u, v)\right](t)\right|=\mid H_{1}\left(t, u_{n}(t), y_{n}(t)\right) \int_{0}^{t} \frac{t}{t+s} F_{1}\left(s, u_{n}(s), v_{n}(s)\right) \mathrm{d} s \\
& -H_{1}(t, x(t), y(t)) \int_{0}^{t} \frac{t}{t+s} F_{1}(s, u(s), v(s)) \mathrm{d} s \\
& \leq H_{1}\left(t, u_{n}(t), y_{n}(t)\right) \int_{0}^{t} \frac{t}{t+s} F_{1}\left(s, u_{n}(s), v_{n}(s)\right) \mathrm{d} s \\
& -H_{1}(t, x(t), y(t)) \int_{0}^{t} \frac{t}{t+s} F_{1}\left(s, u_{n}(s), v_{n}(s)\right) \mathrm{d} s \\
& +H_{1}(t, u(t), y(t)) \int_{0}^{t} \frac{t}{t+s} F_{1}\left(s, u_{n}(s), v_{n}(s)\right) \mathrm{d} s \\
& -H_{1}(t, x(t), y(t)) \int_{0}^{t} \frac{t}{t+s} F_{1}(s, u(s), v(s)) \mathrm{d} s \\
& \leq\left|H_{1}\left(t, u_{n}(t), y_{n}(t)\right)-H_{1}(t, u(t), y(t))\right| \int_{0}^{t} \frac{t}{t+s}\left|F_{1}\left(s, u_{n}(s), v_{n}(s)\right)\right| \mathrm{d} s \\
& +\left|H_{1}(t, x(t), y(t))\right| \int_{0}^{t} \frac{t}{t+s}\left|F_{1}\left(s, u_{n}(s), v_{n}(s)\right)-F_{1}(s, u(s), v(s))\right| \mathrm{d} s \\
& \leq K_{1}\left|H_{1}\left(t, u_{n}(t), y_{n}(t)\right)-H_{1}(t, u(t), y(t))\right|+N_{1} \ln 2 \| F_{1}\left(t, u_{n}(t), v_{n}(t)\right) \\
& -F_{1}(t, u(t), v(t)) \|_{\infty} \text {. }
\end{aligned}
$$

Thus, from the uniform continuity of $H_{1}$, we have $\left\|\mathcal{B}_{1}\left(u_{n}, v_{n}\right)-\mathcal{B}_{1}(u, v)\right\|_{\infty} \rightarrow 0$ as $n \rightarrow \infty$. By carrying out the same steps, we get

$$
\begin{aligned}
\left|\left[\mathcal{B}_{2}\left(u_{n}, v_{n}\right)\right](t)-\left[\mathcal{B}_{2}(u, v)\right](t)\right| \leq & K_{2}\left|H_{2}\left(t, u_{n}(t), y_{n}(t)\right)-H_{2}(t, u(t), y(t))\right|+N_{2} \ln 2 \| F_{2}\left(t, u_{n}(t), v_{n}(t)\right) \\
& -F_{2}(t, u(t), v(t)) \|_{\infty},
\end{aligned}
$$

$\left\|\mathcal{B}_{2}\left(u_{n}, v_{n}\right)-\mathcal{B}_{2}(u, v)\right\|_{\infty} \rightarrow 0$ as $n \rightarrow \infty$. Hence, we have $\left\|\mathcal{B}\left(u_{n}, v_{n}\right)-\mathcal{B}(u, v)\right\| \rightarrow 0$ as $n \rightarrow \infty$. Thus, $\mathcal{B}$ is continuous.

Step 2. Every bounded set $\bar{\Omega}$ in $X, \mathcal{B}(\bar{\Omega})$ is uniformly bounded. Define, $\bar{\Omega}=\left\{(u, v) \in V:\|u\|_{\infty} \leq\right.$ $\left.K^{*}+1,\|v\|_{\infty} \leq K^{*}+1\right\}$, where $K^{*}$ is defined as in Lemma 8 . Let $(u, v) \in \bar{\Omega}$, then we get

$$
\begin{aligned}
\left|\left[\mathcal{B}_{1}(u, v)\right](t)\right| & \leq\left|q_{1}(t)\right|+\mid H_{1}\left(t, u(t), v(t)\left|\int_{0}^{t} \frac{t}{t+s)}\right| F_{1}(s, u(s), v(s) \mid) \mathrm{d} s\right. \\
& \left.\leq W^{q_{1}}+\int_{0}^{t} \frac{t}{t+s} \mid f_{1}(s)\right) \mid \mathrm{d} s \leq W^{q_{1}}+N_{1} K_{1} \leq K^{*}+1 .
\end{aligned}
$$

Similarly, we have

$$
\begin{aligned}
\left|\left[\mathcal{B}_{2}(u, v)\right](t)\right| & \leq\left|q_{2}(t)\right|+\mid H_{2}\left(t, u(t), v(t)\left|\int_{0}^{t} \frac{t}{t+s)}\right| F_{2}(s, u(s), v(s) \mid) \mathrm{d} s\right. \\
& \left.\leq W^{q_{2}}+\int_{0}^{t} \frac{t}{t+s} \mid f_{2}(s)\right) \mid \mathrm{d} s \leq W^{q_{2}}+N_{2} K_{2} \leq K^{*}+1 .
\end{aligned}
$$


Thus, we get

$$
\|\mathcal{B}(u, v)\| \preccurlyeq_{2}\left(\begin{array}{l}
K^{*}+1 \\
K^{*}+1
\end{array}\right) .
$$

Hence, $\mathcal{B}(\bar{\Omega})$ is uniformly bounded in $X$.

Step 3. $\mathcal{B}$ is equicontinuous. For all $t_{1}, t_{2} \in I$ such that $t_{1}<t_{2}$, we get

$$
\begin{aligned}
\left|\left[\mathcal{B}_{1}(u, v)\right]\left(t_{2}\right)-\left[\mathcal{B}_{1}(u, v)\right]\left(t_{1}\right)\right| \leq & \left|q_{1}\left(t_{2}\right)-q_{1}\left(t_{1}\right)\right|+\mid H_{1}\left(t_{2}, u\left(t_{2}\right), v\left(t_{2}\right) \int_{0}^{t_{2}} \frac{t_{2}}{t_{2}+s} F_{1}(s, u(s), v(s) \mathrm{d} s\right. \\
& -H_{1}\left(t_{1}, u\left(t_{1}\right), v\left(t_{1}\right) \int_{0}^{t_{1}} \frac{t_{1}}{t_{1}+s} F_{1}\left(s, u(s), v(s) \mathrm{d} s|\leq| q_{1}\left(t_{2}\right)-q_{1}\left(t_{1}\right) \mid\right.\right. \\
& +\mid H_{1}\left(t_{2}, u\left(t_{2}\right), v\left(t_{2}\right)\right) \int_{0}^{t_{2}} \frac{t_{2}}{t_{2}+s} F_{1}(s, u(s), v(s) \mathrm{d} s \\
& -H_{1}\left(t_{1}, u\left(t_{1}\right), v\left(t_{1}\right)\right) \int_{0}^{t_{2}} \frac{t_{2}}{t_{2}+s} F_{1}(s, u(s), v(s) \mathrm{d} s \mid \\
& +\mid H_{1}\left(t_{1}, u\left(t_{1}\right), v\left(t_{1}\right)\right) \int_{0}^{t_{2}} \frac{t_{2}}{t_{2}+s} F_{1}(s, u(s), v(s) \mathrm{d} s \\
& -H_{1}\left(t_{1}, u\left(t_{1}\right), v\left(t_{1}\right)\right) \int_{0}^{t_{1}} \frac{t_{1}}{t_{1}+s} F_{1}(s, u(s), v(s) \mathrm{d} s \mid \\
\leq & \left|q_{1}\left(t_{2}\right)-q_{1}\left(t_{1}\right)\right|+\mid H_{1}\left(t_{2}, u\left(t_{2}\right), v\left(t_{2}\right)\right) \\
& -H_{1}\left(t _ { 1 } \cdot u \left(t_{1}, v\left(t_{1}\right)\left|\int_{0}^{t_{2}} \frac{t_{2}}{t_{2}+s}\right| F_{1}(s, u(s), v(s) \mid \mathrm{d} s\right.\right. \\
& +\left|H_{1}\left(t_{1}, u\left(t_{1}\right), v\left(t_{1}\right)\right)\right| \mid \int_{0}^{t_{1}} \frac{t_{1}}{t_{1}+s} F_{1}\left(s, u(s), v(s) \mathrm{d} s \mid \cdot F_{2}(s, u(s), v(s) \mathrm{d} s\right. \\
t_{2}+s & \\
& \\
&
\end{aligned}
$$

Since, for all $t, s \in I, t<t+s$, then $1>\frac{t}{t+s}$, therefore, we get

$$
\begin{aligned}
& \mid \int_{0}^{t_{2}} \frac{t_{2}}{t_{2}+s} F_{1}\left(s, u(s), v(s) \mathrm{d} s-\int_{0}^{t_{1}} \frac{t_{1}}{t_{1}+s} F_{1}(s, u(s), v(s) \mathrm{d} s \mid\right. \\
& \quad \leq \int_{0}^{t_{1}} \frac{t_{2}-t_{1}}{t_{1}+s} \mid F_{1}\left(s, u(s), v(s)\left|\mathrm{d} s+\int_{t_{1}}^{t_{2}} \frac{t_{2}}{t_{2}+s}\right| F_{1}(s, u(s), v(s)) \mid \mathrm{d} s\right. \\
& \quad \leq\left|t_{2}-t_{1}\right| \int_{0}^{t_{1}} \frac{1}{t_{1}+s}\left|f_{1}(s)\right| \mathrm{d} s+\int_{t_{1}}^{t_{2}}\left|f_{1}(s)\right| \mathrm{d} s .
\end{aligned}
$$

Then, we have 


$$
\begin{aligned}
\left|\left[\mathcal{B}_{1}(u, v)\right]\left(t_{2}\right)-\left[\mathcal{B}_{1}(u, v)\right]\left(t_{1}\right)\right| \leq & \left|q_{1}\left(t_{2}\right)-q_{1}\left(t_{1}\right)\right|+K_{1}\left|H_{1}\left(t_{2}, u\left(t_{2}\right), v\left(t_{2}\right)\right)-H_{1}\left(t_{1}, u\left(t_{1}\right), v\left(t_{2}\right)\right)\right| \\
& +N_{1}\left[\left|t_{2}-t_{1}\right| \int_{0}^{t_{1}} \frac{1}{t_{1}+s}\left|f_{1}(s)\right| \mathrm{d} s+\int_{t_{1}}^{t_{2}}\left|f_{1}(s)\right| \mathrm{d} s\right] .
\end{aligned}
$$

Similarly, we obtain that

$$
\begin{aligned}
\left|\left[\mathcal{B}_{2}(u, v)\right]\left(t_{1}\right)-\left[\mathcal{B}_{2}(u, v)\right]\left(t_{2}\right)\right| \leq & \left|q_{2}\left(t_{2}\right)-q_{2}\left(t_{1}\right)\right|+K_{2}\left|H_{2}\left(t_{2}, u\left(t_{2}\right), v\left(t_{2}\right)\right)-H_{2}\left(t_{1}, u\left(t_{1}\right), v\left(t_{2}\right)\right)\right| \\
& +N_{2}\left[\left|t_{2}-t_{1}\right| \int_{0}^{t_{1}} \frac{1}{t_{1}+s}\left|f_{2}(s)\right| \mathrm{d} s+\int_{t_{1}}^{t_{2}}\left|f_{2}(s)\right| \mathrm{d} s\right] .
\end{aligned}
$$

Thus, when $t_{1} \rightarrow t_{2},\left|\left[\mathcal{B}_{1}(u, v)\right]\left(t_{1}\right)-\left[\mathcal{B}_{1}(u, v)\right]\left(t_{2}\right)\right| \rightarrow 0$ and $\left|\left[\mathcal{B}_{2}(u, v)\right]\left(t_{1}\right)-\left[\mathcal{B}_{2}(u, v)\right]\left(t_{2}\right)\right| \rightarrow 0$. Thus, $\mathcal{B}(\bar{\Omega})$ is equicontinuous on $I$. So, it follows that $\mathcal{B}(\bar{\Omega})$ is relatively compact. Thus, $\mathcal{B}: \bar{\Omega} \rightarrow X$ is completely continuous.

Theorem 10. Let conditions (C1)-(C6) hold and assume that the following condition also holds:

(C7) $\sigma(T)<1$, where

$$
T=\left(\begin{array}{ll}
\frac{(\psi(1)-\psi(0))^{\alpha}}{\Gamma(\alpha+1)} p_{11}\left(W^{q_{1}}+N_{1} K_{1}\right) & \frac{(\psi(1)-\psi(0))^{\alpha}}{\Gamma(\alpha+1)} p_{12}\left(W^{q_{2}}+N_{2} K_{2}\right) \\
\frac{(\psi(1)-\psi(0))^{\beta}}{\Gamma(\beta+1)} p_{21}\left(W^{q_{1}}+N_{1} K_{1}\right) & \frac{(\psi(1)-\psi(0))^{\beta}}{\Gamma(\beta+1)} p_{22}\left(W^{q_{2}}+N_{2} K_{2}\right)
\end{array}\right) .
$$

Then the proposed system (7) has at least one solution in $X$.

Proof. We proved that $\mathcal{A}$ is contractive and $\mathcal{B}: \bar{\Omega} \rightarrow X$ is completely continuous. Now, let $(u, v) \in X$ be a solution of

$$
(u, v)=\lambda \mathcal{A}\left(\frac{u}{\lambda}, \frac{v}{\lambda}\right) \mathcal{B}(u, v),
$$

then $\|u\| \leq K^{*},\|v\| \leq K^{*}$. Thus, the operator $\mathbb{T}$ has a fixed point $t \rightarrow\left(u^{*}(t), v^{*}(t)\right) \in X$ and this fixed point is the solution of system (7).

The next theorem is a direct result of Theorem 10 when $\psi(t)=t^{\theta}$ for some $\theta \in(0, \infty)$.

Theorem 11. Let conditions $(C 1)-(C 7)$ hold and $\theta \in(0, \infty)$. Then the following system has at least one solution

$$
\begin{aligned}
u(t)= & \left(p_{1}(t)+\frac{1}{\Gamma(\alpha)} \int_{0}^{t} \theta s^{\theta-1}\left(t^{\theta}-s^{\theta}\right)^{\alpha-1} V_{1}(s, u(s), v(s)) \mathrm{d} s\right) \\
& \times\left(q_{1}(t)+H_{1}(t, u(t), v(t)) \int_{0}^{t} \frac{t}{t+s} F_{1}(s, u(s), v(s)) \mathrm{d} s\right), \quad t \in[0,1], \alpha \in(0,1), \\
v(t)= & \left(p_{2}(t)+\frac{1}{\Gamma(\beta)} \int_{0}^{t} \theta s^{\theta-1}\left(t^{\theta}-s^{\theta}\right)^{\beta-1} V_{2}(s, u(s), v(s)) \mathrm{d} s\right) \\
& \times\left(q_{2}(t)+H_{2}(t, u(t), v(t)) \int_{0}^{t} \frac{t}{t+s} F_{2}(s, u(s), v(s)) \mathrm{d} s\right), \quad t \in[0,1], \beta \in(0,1) .
\end{aligned}
$$

The next corollary can be proved by setting $\theta=1$ in the previous theorem. 
Corollary 12. Let conditions (C1)-(C7) hold. Then the following system has at least one solution

$$
\begin{aligned}
u(t)= & \left(p_{1}(t)+\frac{1}{\Gamma(\alpha)} \int_{0}^{t}(t-\zeta)^{\alpha-1} V_{1}(\zeta, u(\zeta), v(\zeta)) \mathrm{d} \zeta\right) \\
& \times\left(q_{1}(t)+H_{1}(t, u(t), v(t)) \int_{0}^{t} \frac{t}{t+\zeta} F_{1}(\zeta, u(\zeta), v(\zeta)) \mathrm{d} \zeta\right), \quad t \in[0,1], \alpha \in(0,1), \\
v(t)= & \left(p_{2}(t)+\frac{1}{\Gamma(\beta)} \int_{0}^{t}(t-\zeta)^{\beta-1} V_{2}(\zeta, u(\zeta), v(\zeta)) \mathrm{d} \zeta\right) \\
& \times\left(q_{2}(t)+H_{2}(t, u(t), v(t)) \int_{0}^{t} \frac{t}{t+\zeta} F_{2}(\zeta, u(\zeta), v(\zeta)) \mathrm{d} \zeta\right), \quad t \in[0,1], \beta \in(0,1) .
\end{aligned}
$$

\section{Conclusion}

In this article, a nonlinear merged system of Urysohn Volterra-Chandrasekhar integral equations was investigated in generalized Banach algebras. We obtained the solvability of the proposed system by applying the new combined Leray-Schauder with Perov's fixed point approach.

\section{References}

[1] J. Banas, M. Jleli, M. Mursaleen, B. Samet, and C. Vetro, Advances in Nonlinear Analysis via the Concept of Measures of Noncompactnes, Springer, Singapore, 2017.

[2] K. Deimling, Nonlinear Functional Analysis, Springer, Berlin, 1985.

[3] P. P. Zabrejko, A. I. Koshelev, M. A. Krasnosel'skii, S. G. Mikhlin, L. S. Rakovschik, and V. J. Stetsenko, Integral Equations, Nordhoff, Leyden, MA, USA, 1975.

[4] M. A. Polo-Labarrios, S. Q. Garcia, G. E. Paredes, L. F. Perez, and J. O. Villafuerta, Novel numerical solution to the fractional neutron point kinetic equation in nuclear reactor dynamics, Ann. Nucl. Energy 137 (2020), 10717, DOI: 10.1016/ j.anucene.2019.107173.

[5] H. Chen, J. I. Frankel, and M. Keyhani, Two-probe calibration integral equation method for nonlinear inverse heat conduction problem of surface heat fluxestimation, Int. J. Heat Mass Transf. 121 (2018), 246-264, D0I: 10.1016/ j.ijheatmasstransfer.2017.12.072.

[6] T. E. Roth and W. C. Chew, Stability analysis and discretization of A- $\Phi$ time domain integral equations for multiscale electromagnetic, J. Comput. Phys. (2019), 109102, DOI: 10.1016/j.jcp.2019.109102.

[7] E. Cuesta, M. Kirance, and S. A. Malik, Image structure preseving denoising generalized fractional time integrals, Signal Process. 92 (2012), 553-563.

[8] J. Banas and A. Chlebowicz, On a quadratic integral equation of Erdelyi-Kiber type in the class of subpower functions, J. Nonlinear Convex Anal. 19 (2018), 823-840.

[9] J. Banas and A. Dubiel, Solvability of a Volterra-Stieltjes integral equations in the class of functions having limits at infinity, Electr. J. Qualit. Th. Diff. Equations 53 (2017), 1-17, DOI: 10.14232/ejqtde.2017.1.53.

[10] J. R. Wang, C. Zhu, and M. Feckar, Solvability of fully nonlinear functional equations involving Erdelyi-Kober fractional integrals on the unbounded interval, Optimization 63 (2014), no. 8, 1235-1248, DOI: 10.1080/02331934.2014.883513.

[11] R. Hilfer, Applications of Fractional Calculs in Phyiscs, World Scientific, New York, 2000.

[12] R. Almeida, A Caputo fractional derivative of a function with respect to another function, Commun. Nonlinear Sci. Numer. Simul. 44 (2017), 460-481, DOI: 10.1016/j.cnsns.2016.09.006.

[13] M. A. Darwish, J. R. Graef, and K. Sadarangan, On Urysohn-Volterra fractional quadratic integral equations, J. Appl. Anal. Comput. 8 (2018), no. 1, 331-343, DOI: 10.11948/2018.331.

[14] J. J. Nieto, A. Ouahab, and R. Rodriguez-Lopez, Fixed point theorems in generalized Banach Algebras and applications, Fixed Point Theory 19 (2018), no. 2, 707-732, DOI: 10.24193/fpt-ro.2018.2.54. 
[15] H. Hashem, A. El-Sayed, and D. Baleanu, Existence results for block matrix operator of fractional orders in Banach algebras, Mathematics 7 (2019), 856, DOI: 10.3390/math7090856.

[16] A. B. Amar, A. Jeribi, and B. Krichen, Fixed point theorems for block matrix and an applications to a structured problem under boundary conditions of Rotenberg's model type, Math. Slovaca 64 (2014), 155-174, DOI: 10.2478/s12175-0130193-3.

[17] S. Chandrasekhar, Radiative Transfer, Oxford University Press, London, 1950 and Dover Publication, New York, 1960.

[18] J. Banas, M. Lecko, and W. G. El-Sayed, Existence theorem for some quadratic integral equations, J. Math. Anal. Appl. 222 (1998), 276-286.

[19] H. H. Hashem and A. M. A. El-Sayed, Stabilization of coupled systems of quadratic integral equations of Chandrasekhar type, Math. Nachr. 290 (2017), no. 2-3, 341-348, DOI: 10.1002/mana.201400348.

[20] D.-Chen Chang and S.-Ya Feng, On integral equations of Chandrasekhar type, J. Nonlinear Convex Anal. 19 (2018), no. 3, 525-541.

[21] A. Jeribi, N. Naddachi, and B. Krichen, Fixed-point theorems for multivalued operator matrix under weak topology with an applications, Bull. Malays. Math. Sci. Soc. 43 (2020), 1047-1067, DOI: 10.1007/s40840-019-00724-w.

[22] H. H. G. Hashem, Solvability of a $2 \times 2$ block operator matrix of Chandrasekhar type on a Banach algebra, Filomat 31 (2017), no. 16, 5169-5175, DOI: 10.2298/FIL1716169H.

[23] A. Jeribi, N. Kaddachi, and B. Krichen, Fixed point theorem of block operator matrices on Banach algebra and an application to functional integral equations, Math. Methods Appl. Sci. 36 (2013), no. 6, 659-673, DOI: 10.1002/ mma.2615.

[24] R. Arab and M. Mursaleen, On existence of solution of a class of quadratic-integral equations using contraction defined by simulation functions and measure of noncompactness, Carpathian J. Math. 34 (2018), no. 3, 371-378.

[25] R. Arab, M. Mursaleen, and S. M. H. Rizvi, Positive solution of a quadratic integral equation using generalization of Darbo's fixed point theorem, Numer. Funct. Anal. Optim. 40 (2019), no. 10, 1150-1168, DOI: 10.1080/ 01630563.2019 .1589496$.

[26] A. Jeribi, B. Krichen, and B. Mefteh, Existence of solutions for an infinite system of qadratic integral equations in the Banach algebra $C\left(I, c_{0}\right)$, Mediterr. J. Math. 17 (2020), 10, DOI: 10.1007/s00009-019-1460-5.

[27] G. Darbo, Punti uniti in transformazioni a condominio non compalto, Rend. Sem. Math. Univ. Padova 24 (1955), 84-92.

[28] M. Cvetkovic and V. Rakocevic, Extensions of Perov theorem, Carpathian J. Math. 31 (2015), no. 2, 181-188.

[29] M. Cvetkovic, On the eqivalence between Perov fixed point theorem and Banach contraction principle, Filomat 31 (2017), no. 11, 3137-3146, DOI: 10.2307/26195042.

[30] A. I. Perov, On the Cauchy problem for a system of ordinary differential equations, Pviblizhen. Met. Reshen. Differ. Unavn. 2 (1964), 115-134. 